\title{
Zur Entstehung des Talkessels von La Paz/Bolivien und Umgebung
}

\section{Einleitung}

Die Stadt La Paz erstreckt sich in einer $3-6 \mathrm{~km}$ breiten Schlucht am nordöstlichen Rand des bolivianischen Altiplano. Sie wächst teilweise auf diese Hochebene hinauf und nimmt damit Höhenlagen zwischen ca. 3250 und $4100 \mathrm{~m}$ ein (Fig.1).

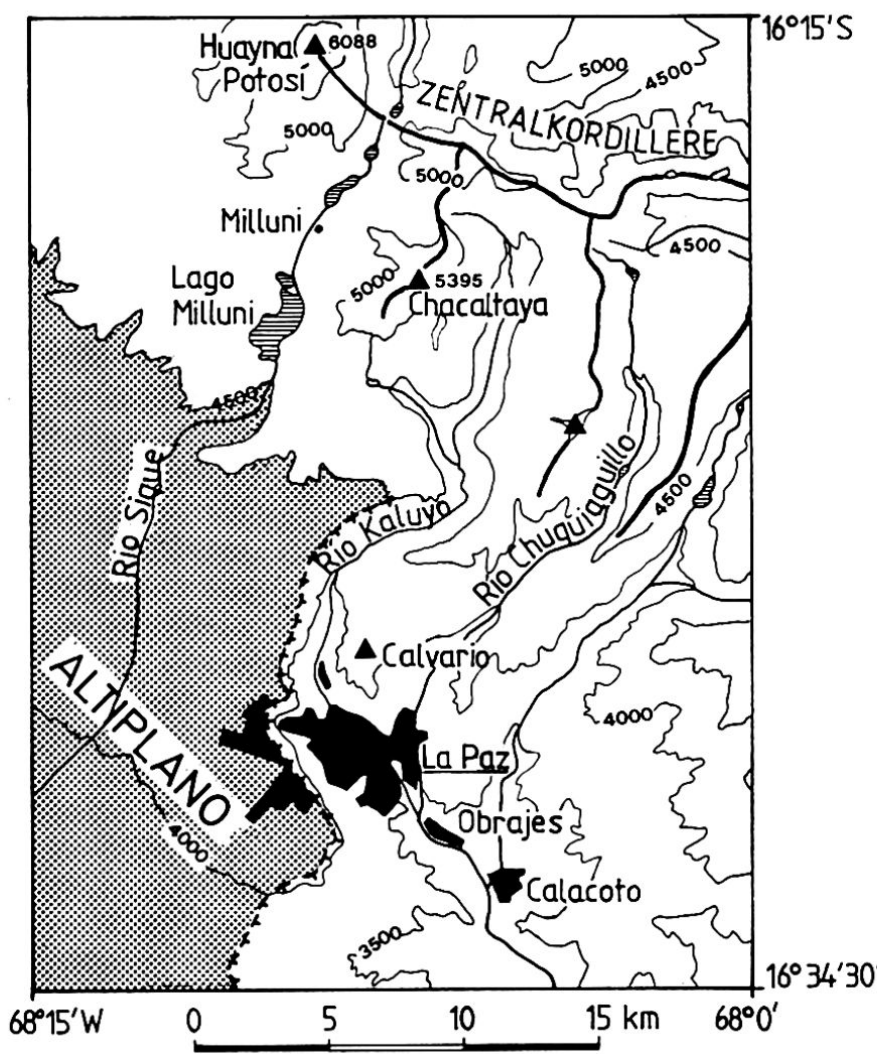

Fig.1: Lage der Stadt La Paz zwischen Altiplano und Zentralkordillere

Die Entstehung der Schlucht ist Hauptgegenstand der vorliegenden Arbeit. Detaillierte Studien gehen auf TROLL \& FINSTERWALDER (1935), AHLFELD $(1946,1972)$ und DOBROVOLNY (1962) zurück. Neuere Arbeiten stammen von SERVANT (1977).

1) Die "Formación La Paz" ist nach DOBROVOLNY (1962, S.20 f.) dreiteilig und wird in eine untere, mittlere und obere Einheit unterteilt.
Aufschlüsse von Lockermaterial sind im ganzen Stadtgebiet und in den Tälern der Umgebung häufig. Fels tritt dagegen nur ausserhalb der Stadt zutage, und zwar sind es vorwiegend paläozoische Schiefer und rote, kretazische Sandsteine (AHLFELD 1972, S.92). Wie mächtig die darauf abgelagerten Sedimente in $\mathrm{La} \mathrm{Paz}$ sind, ist noch nicht mit Bohrungen ermittelt worden. Es kann aber aus der grossen Tiefe der Schlucht geschlossen werden, dass ursp: ünglich überall mindestens $500 \mathrm{~m}$ Schotter und Moränen angehäuft gewesen sind. Das Hauptproblem besteht darin, dieses Lockermaterial und die entsprechenden Oberflächenformen zu datieren und so die Abfolge ihrer Formungsprozesse herzuleiten.

\section{Möglichkeiten der Datierung}

\subsection{Fossilien}

In der Literatur finden sich nur spärliche Hinweise auf Vorkommen fossiler Säugetiere in den Sedimenten der Formation la Paz 1). So erwähnt POSNANSKY (1920, S.97) Funde von Scelidotherium und Parahipparion aus dem Stadtteil Miraflores. RADELLI (1964, S.842) beschreibt Vorkommen von Xotodon sp. und Stenotephanos sp., und schliesslich sind Angaben bei VILLARROEL (1977, S.30) über Posnanskytherium desaguaderoi. Diese Funde stamen aus dem Stadtteil Obrajes/Al to Seguëncoma (16032'S/68 $\left.6^{\circ} 30^{\prime \prime} \mathrm{W}, 3430 \mathrm{~m}\right)$ und umfassen einen Teil eines horizontalen Mandibelasts (Foto 1), einzelne obere und untere Molaren (Foto 2) und das distale Ende eines Femur (Foto 3). In unmittelbarer Nähe dieser Lokalität sind weitere Fossilien zum Vorschein gekommen. Ein Teil ei" nes horizontalen Asts einer Mandibel mit fehlenden Zähnen sowie ein drittes Mctacarpale (?) gehören zu einem kleinen Macrauchenidae. Im weiteren stammt ein Stück Panzer von einem Glyptodontidae, und zwar der Subfamilie Sclerocalyptinae (Foto 4).

Zum ersten Literaturhinweis bei POSNANSKY (1920) muss beigefügt werden, dass die erwähnten Fossilien nicht durch Illustrationen belegt sind. Wir können daher kaum schlüssige Folgerungen dar-

Dr. Carlos Villarroel, Servicio Geológico de Bolivia, Casilla 2729, La Paz.

PD Dr. Kurt Graf, Oberassistent am Geographischen Institut der Universität Zürich, Blümlisalpstr.10, 8006 Zürich. 


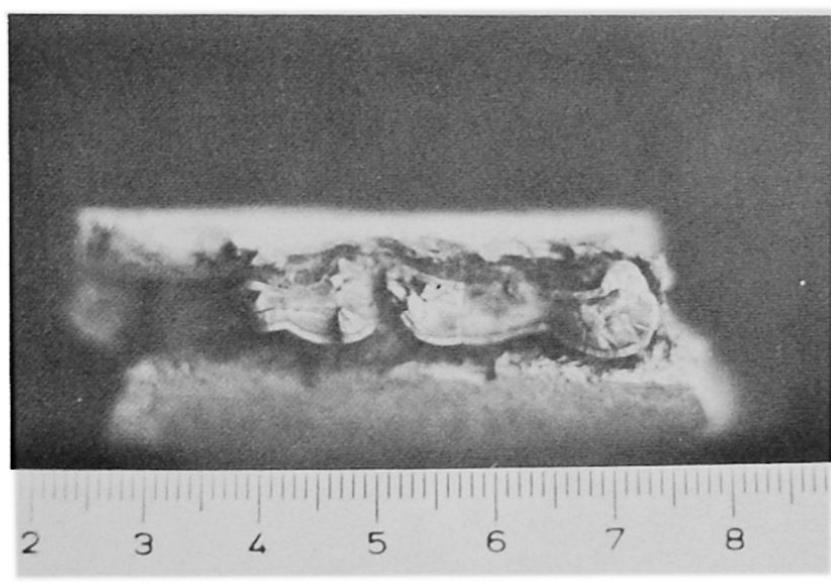

Foto 1: Teil eines horizontalen Mandibelasts von Posnanskytherium desaguaderoi aus den oberpljozänen Schottern von La Paz (16 32 'S/ $68^{\circ} 6^{\prime} 30^{\prime}(\mathrm{W}, 3430 \mathrm{~m}$ )

aus ziehen, bevor nicht neue Funde diese Identifikationen bestätigen oder widerlegen. Wenigstens 1iegen unsere oben aufgeführten Fossilien vor, welche wie jene bei POSNANSKY aus der mittleren Einheit der Formation La Paz stammen. Sie lassen als sehr unwahrscheinlich erscheinen, dass die mittlere Einheit quartäre Säugetierreste enthält, wie sie Scelidotherium und Parahipparion darstellen.

Als Ergänzung zu RADELLI (1964) haben wir in einer früheren Arbeit (VILLARROEL 1977, S.29) bereits ausgeführt, dass Xotodon und Stenotephanos mit grosser Wahrscheinlichkeit zu Posnanskytherium zählen. Dabei handelt es sich um den einzigen Toxodonten, der bis heute in den Schottern der Formation La Paz gefunden worden ist. Möglicherweise ist RADELLI bei den Identifikationen fehlgeleitet worden, da er sie nur aufgrund von oberen Molaren durchgeführt hat. Diese sind aber bei allen drei Gattungen recht ähnlich.

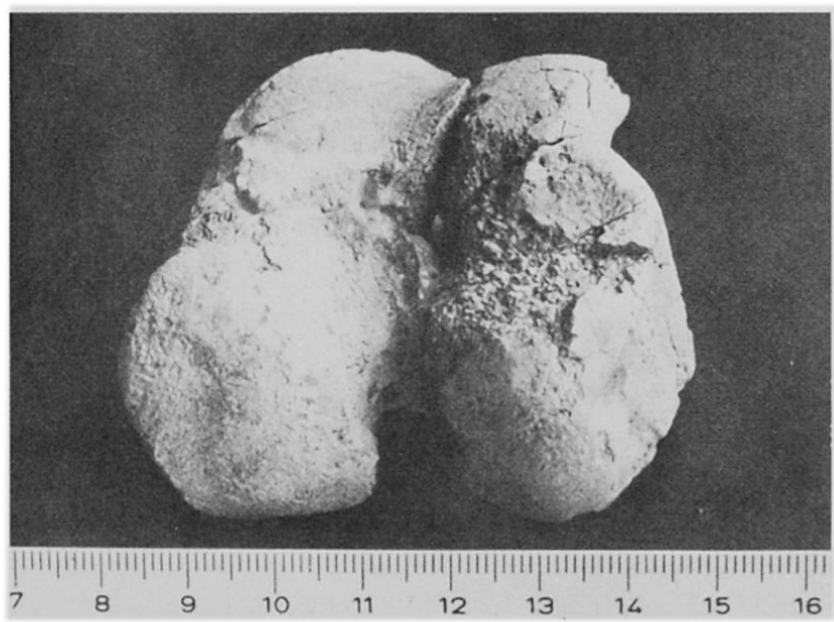

Foto 3: Distales Ende eines Femur (entspr. Foto 1)

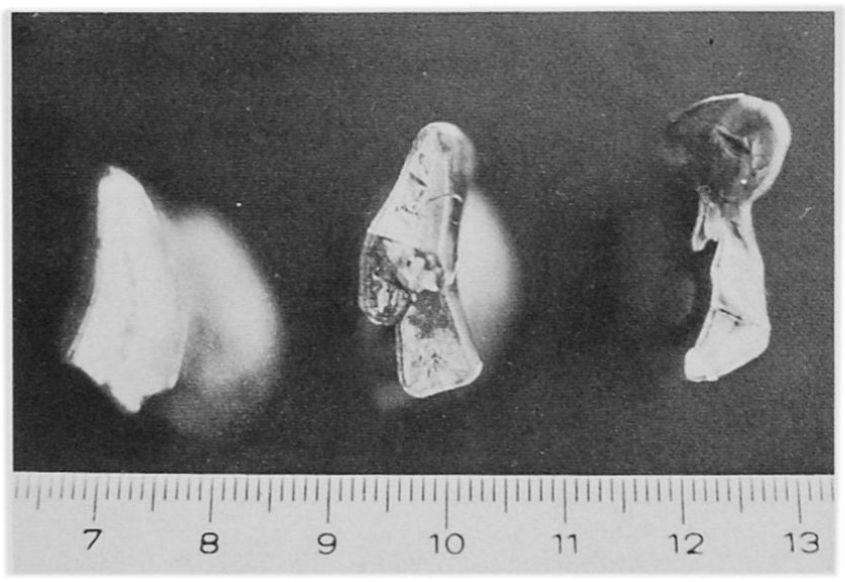

Foto 2: Einzelne Molaren (entspr. Foto 1)

Posnanskytherium stellt ein charakteristisches Fossil aus dem Oberpliozän dar. Dies kann nicht nur aus seinem Evolutionsgrad abgeleitet werden, sondern vor allem aus der ausgiebig dokumentierten, gleichaltrigen Fauna, die zusammen mit Posnanskytherium in Sedimenten der Formation Umala bei den Lokalitäten Ayo Ayo - Vizcachani (170 5-10'S/67055'-680W) und Umala (17020'S/680W) gefunden worden sind. Wir dürfen also annehmen, dass die Schotter der Formation La Paz oder zumindest ihre mittlere und obere Einheit im Oberpliozän geschüttet worden sind. Sehr wahrscheinlich ist ihre Sedimentation kontinuierlich erfolgt, und dies in der ganzen Abfolge bis zur Diskordanz an ihrer Obergrenze. Dort sind 1-15 m vulkanischer Tuff aufgelagert ("Toba Chijini" nach DOBROVOLNY 1962, S.32; CLOCHIATTI et al. 1978, S.130), welcher die Grenze zwischen Oberpliozän und Quartär markiert (s. Kap. 2.4.). Mit der Diskordanz könnte man die letzte grosse Hebungsphase der andinen Orogenese korrelieren. Aufgrund von Vergleichen zwischen der pliozänen und aktuellen Fauna ist anzunehmen, dass mit dieser letzten Phase eine Hebung von mindestens $1000 \mathrm{~m}$ erfolgt ist.
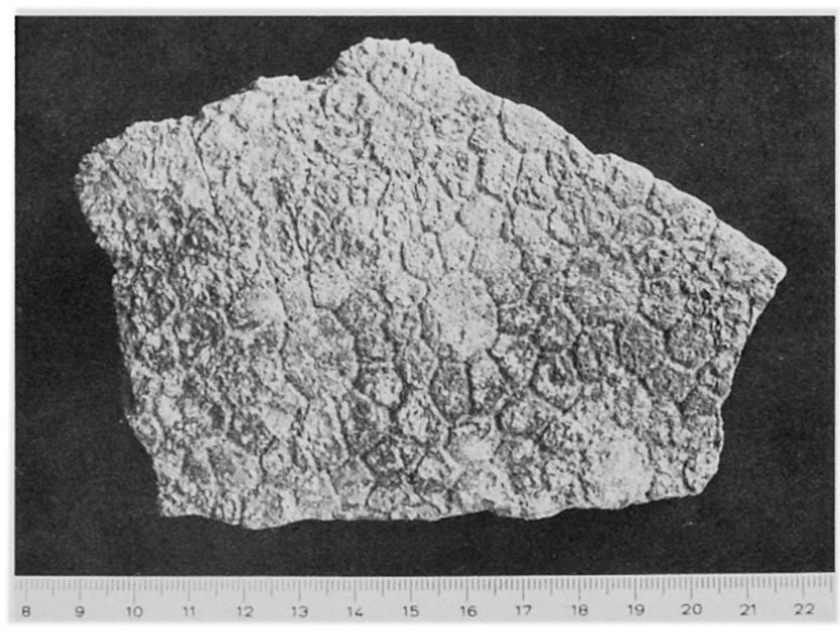

Foto 4: Stück eines Panzers von einem Vertreter der Glyptodontidae, Subfamilie Sclerocalyptinae 


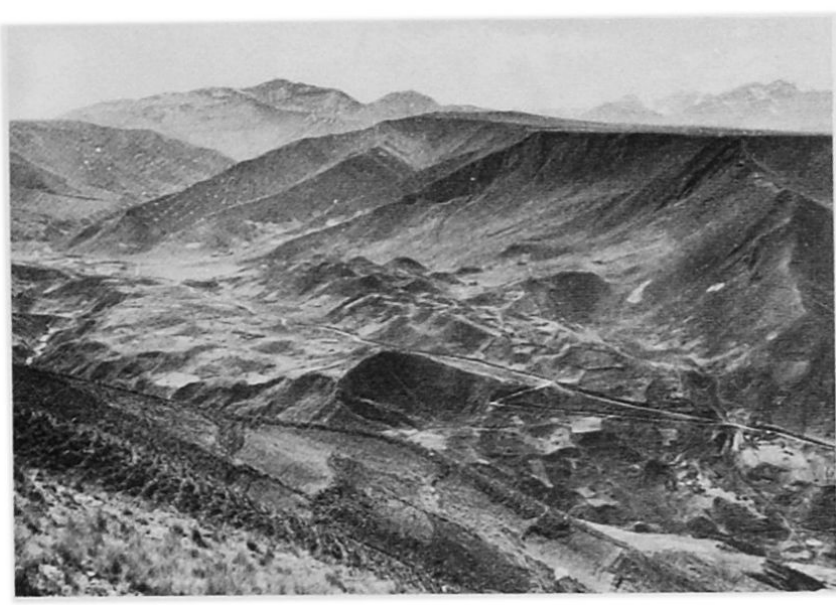

Foto 5: Blick von SW auf das sitiplano und das Tal des R10 Kaluyo. Das gewellte Relief in der Bildmitte bilden Endmoränenwälle vom Maximalstand der letzten Eiszeit.

\subsection{Pollenanalyse}

Eine zweite Datierungsmöglichkeit betrifft die Pollenanalyse. Insbesondere geht es darum, typisch tertiäre Pollenkörner zu finden. Als eine Möglichkeit ist der vulkanische Tuff untersucht worden, der auf 3830 - 3900 m einen Leithorizont darstellt. Seine Hauptbestandteile sind nach DOBROVOLNY (1962, S.34) 71,5\% $\mathrm{SiO}_{2}, 13,5^{\circ} \mathrm{Al}_{2} \mathrm{O}_{3}$ und $5 \% \mathrm{~K} 2 \mathrm{O}$. Die palynologische Präparation ist prinzipiell möglich, indem die Tuffproben mit HFkonz. gekocht und mindestens zehnmal mit warmer $\mathrm{HCl}_{\text {konz }}$. behandelt werden. Nach der Flusssäure darf aber bei keinem Arbeitsschritt mehr Wasser verwendet werden, da sonst ein unlösliches, weisses Gel entsteht (Silicogele oder $\mathrm{CaF}_{2}$ ?). Tuffproben aus fünf Lokalitäten von La $\mathrm{Paz}$ sind analysiert worden, doch die organischen Rückstände reichen nirgends für eine pollenanalytische Auswertung. Entweder sind die Pollenkörner seit ihrer Einbettung einer zu starken mechanischen oder chemischen Verwitterung ausgesetzt gewesen, oder dann ist der Tuff in einer

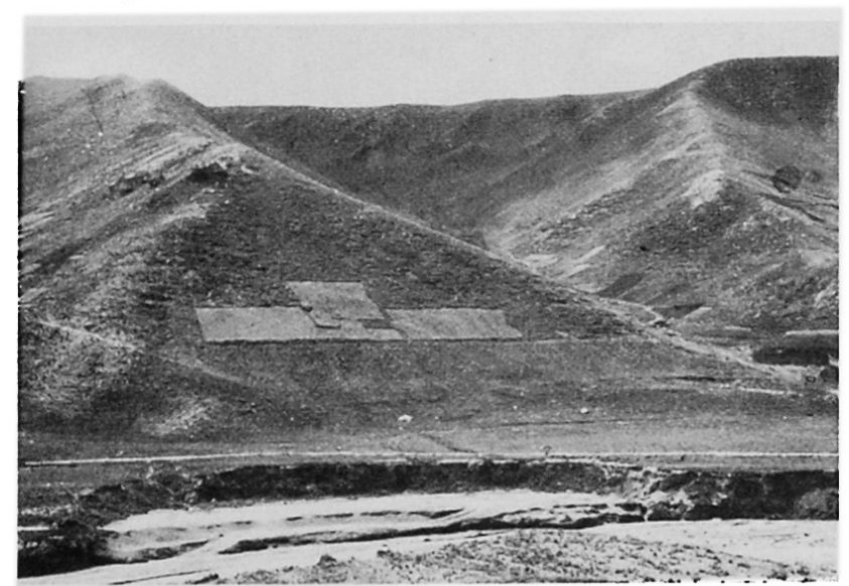

Foto 6: Der R10 Kaluyo unterschneidet am Ort der Probenentnahme seine Niederterrasse (16 $26^{\prime} \mathrm{S} /$ $\left.68^{\circ} 8^{\prime} \mathrm{W}, 4070 \mathrm{~m}\right)$. Das aufgeschlossene Profil (Fig.2) weist vorwiegend Schotter auf, dazwischen aber auch datierbare Torfhorizonte bis 9645 BP. Oberhalb der Aecker im Hintergrund ist ein Felsband aus vulkanischem Tuff erkennbar. so kurzfristigen Eruption abgelagert worden, dass die Pollendichte zum voraus gering ausgefallen ist.

Pollenanalysen sind uns lediglich von postglazialen Proben gelungen. Zur Illustration zeigen wir ein unvollständiges Profil von einer Niederterrasse an Río Kaluyo, wenig oberhalb La Paz (Fig. 2) .

Alle Proben enthalten zur Hauptsache Sporomorphen von Pteridophyta, Gramineae und Compositae. Damit sind sie weitgehend durch Sporomorphen belegt, die möglicherweise durch den wind hierher transportiert worden sind. Das gleiche gilt für die einzigen Baumpollenkörner (Podocarpus und Alnus). Immerhin kann man im Pollendiagramm Tendenzen feststellen, dass im unteren Diagrammabschnitt mehr Podocarpus, oben dagegen mehr Umbelliferae, Chenopodiaceae und Amaranthaceae auftreten. Aufgrund dieser Angaben erweist sich aber eine altersmässige Differenzierung mit Hilfe der Pollenanalyse auch bei postglazialen Proben als problematisch.

\subsection{Absolute Datierungen}

Eine weitere Möglichkeit zur Datierung besteht in der Anwendung von radiometrischen Methoden. Dazu bemerken EVERDEN et a1. (1977, Abb.1), dass eine Datierung des Tuffs "Chijini" mit der K-ArMethode ein Alter von $6 \mathrm{Mi} 11$. y ergeben habe. Dieses Alter ist indessen nach unseren paläontologischen Befunden zu hoch. Möglicherweise liegt der Fehler bei den datierten Biotiten, da sie durchaus als sekundäre Ablagerungen in den Tuff gelangt sein können. Dies wird von CLOCCHIATTI et al. (1978, S.129) bestätigt, indem er den Tuff als pyroklastisch-klastische Bildung ansieht und nicht ausschliesslich als pyroklastisch (d.h. vulkanisch), wie das früher stets angenommen worden ist.

Mit Hilfe der ${ }^{14} \mathrm{C}$-Methode ergab sich das Mindestalter mehrerer Torfmoore und anderer feuchter Lokalitäten, die eiszeitlich vergletschert waren. Die Datierungen streuen zwischen $610 \mathrm{BP}$ und $9800 \mathrm{BP} 2$ ) und stecken damit wohl die unge-

\footnotetext{
2) Folgende Datierungen von Basisproben aus dem Raum La Paz sind im Niedersächsischen Landesamt für Bodenforschung in Hannover durchgeführt

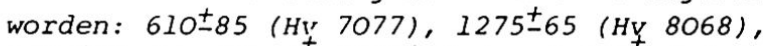
$2470 \pm 95(\mathrm{HV} 7090)^{+}, 3525 \pm 95(\mathrm{HV} 8078)^{+}, 5050 \pm 50$ $(H V 8080), 5425 \pm 60(H V 8070), 6440 \pm 75$ ( $H V$ 6217), $7310 \pm 380(\mathrm{HV} 7080), 9385 \pm 120\left(H_{V} 7088\right), 9645 \pm$ $90(\mathrm{HV} 8073)$ und $9800 \pm 100 \mathrm{BP}$ ( HV 8529).
}

siehe nächste Seite

Fig.2: Pollendiagramm von einer Schwemmebene hinter den Endmoränenwällen im Tal des R10 Kaluyo $\left(16^{\circ} 26^{\prime} \mathrm{S} / 68^{\circ} 8^{\prime} \mathrm{W}, 407 \mathrm{~m}\right)$. Darin bedeuten SPS die sporomorphensumme (=100\% inkl.Gramineae und Ptegridophyta), SPF die Sporomorphenfrequenz pro $\mathrm{cm}^{2}, \mathrm{D} 1$ und $\mathrm{D} 2$ zwei Diagrammabschnitte. Indet.No.1 ist wahrscheinlich eine Megaspore, bei Perezia handelt es sich um eine Gattung der Compositae tubuliflorae vom Tribus Mutisieae. 
Fig. 2 Profil Río Kaluyo

$16^{\circ} 26^{\prime} \mathrm{S} / 68^{\circ} 8^{\prime} \mathrm{W}, 4070 \mathrm{~m}$; K.Graf

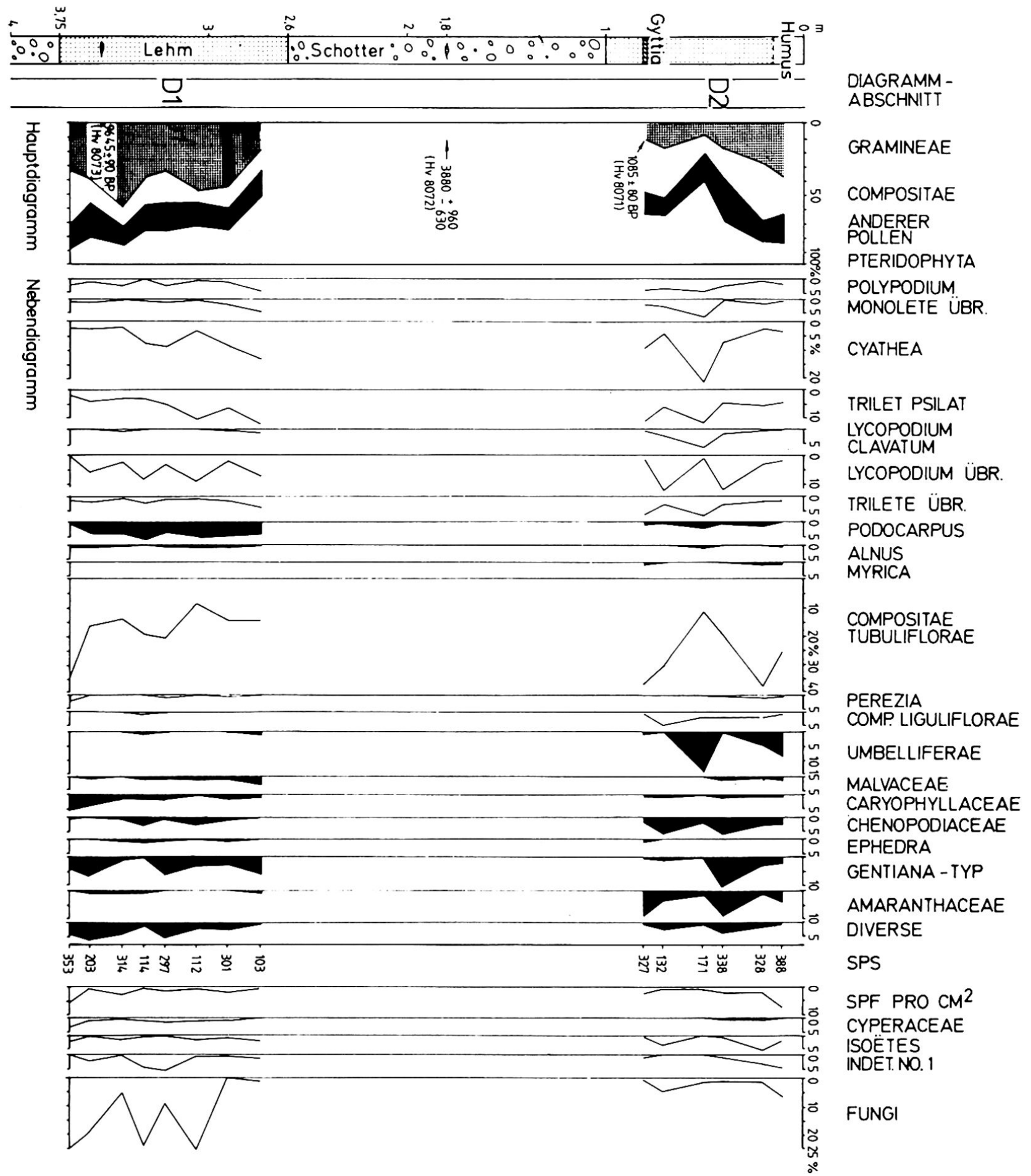




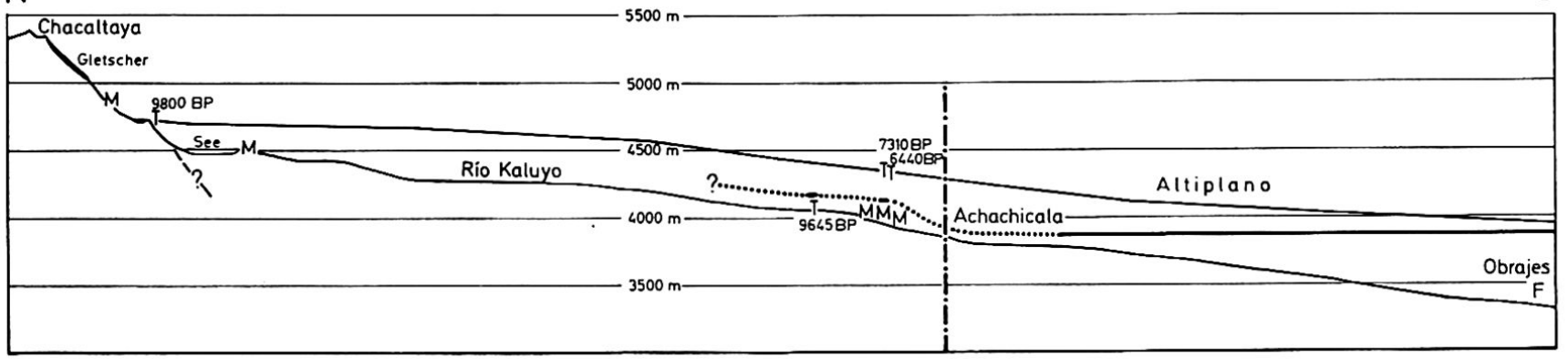

Fig.3: Geomorphologisches Längsprofil, das dem R1o Kaluyo folgt. Die Ausdehnung der Stadt La Paz ist mit den Quartieren Achachicala und Obrajes abgesteckt. Mit M sind Endmoränen bezeichnet, mit $T$ datierte Torfmoore und mit $F$ unser Fossilienfundort. Zwischen Talboden und Horizontlinie (dünne Linien) verläuft das Tuffband (dicke Linien, bei Unsicherheit punktiert). Die morphogenetische Altiplanogrenze ist strichpunktiert.

Aahre Dauer des Postglazials in Bolivien ab. Ueberdies zeigen sie, dass während des Postglazials nie Gletscherzungen in den Tälern unterhalb ca. $4700 \mathrm{~m}$ gestirnt haben.

\subsection{Vergleichende Feldbeobachtungen}

Als vierte Datierungsmöglichkeit zichen wir vergleichende Feldbeobachtungen bei. So wollen wir beispielsweise die Ausdehnung der letzteiszeitlichen Gletscher im Raum La Paz festlegen. Da in der Schlucht viele Vorzeitformen zerstört worden sind, ergeben sich dabei Schwierigkeiten. Die untersten, deutlich ausgebildeten Moränenwälle liegen hier auf ca. $4000 \mathrm{~m}$ (Fig.3 und 4, Foto 5), aber vielleicht sind ticferliegende bereits unkenntlich oder wegerodiert. Zum Vergleich betrachten wir daher die untersten Moränenwälle an anderen Stellen des Altiplano, die weniger starken Abtragungsprozessen ausgesetzt sind. Sie liegen im Titicacaseeraum auf $4050 \mathrm{~m}$ (Halbinsel Copacabana, GRAF 1975, S.11/12) und ca. $4200 \mathrm{~m}$ (östlich von Rosapata, NEWELL 1949, S.86) 3). Im südlichen Altiplano reichen Moränenwälle maximal bis $3950 \mathrm{~m}$ (Cerro Azanaques, GRAF 1975, S.11/12). Mit grosser Wahrscheinlichkeit können wir alle diese Moränen miteinander korrelieren, einschliesslich jener von $\mathrm{La} \mathrm{Paz}$, und sie dem Maximum der letzten Eiszeit zuordnen. Weitere grossräumige Analogieschlüsse ziehen wir bei-der Betrachtung der Tuffschicht in La Paz (s.Fig.3 und 4, Foto 6). Sie zeichnet eine mehr oder weniger horizontale Verebnungsfiäche auf $3830-3900 \mathrm{~m}$ nach, welche vor der Tuffablagerung angelegt worden ist. Die Höhenlage dieser Peneplain stimmt recht genau mit jener des nördlichen Altiplano überein, sofern wir nur den eiszeit lich unvergletscherten Streifen südwestlich von La Paz herausgréifen. Eine Peneplainisierung dieses Ausmasses muss während einer viel längeren Zeit der Akkumulation und Denudation ausgesetzt gewesen sein, als dies im Pleistozän möglich gewesen ist. Folglich reicht die Hauptanlage dieser Ebene ins Pliozän zurück. Wann aber ist dieses Relief im Gebiet von La Paz fossilisiert worden, am Ende des Pliozäns oder im Altpleistozän? Wir stellen fest, dass randlich der

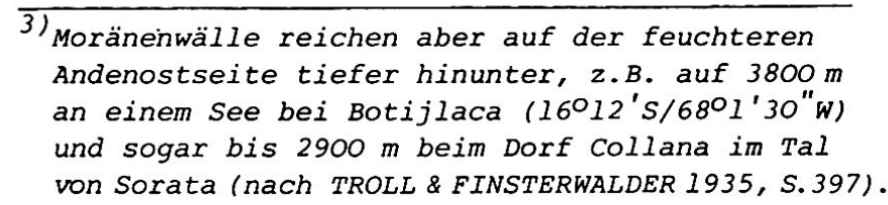

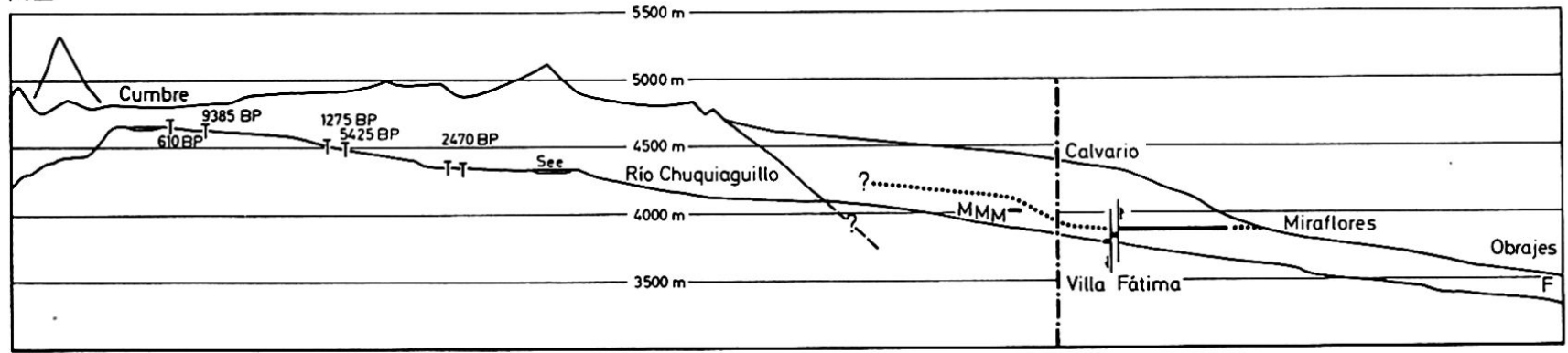

0 $5 \mathrm{~km}$

Fig.4: Geomorphologisches Längsprofil, das dem R1o Chuquiaguillo folgt. Die eingetragenen Stadtviertel sind hier Villa Fatima, Miraflores und Obrajes. Zur Legende siehe Text von Fig. 3 . 
Peneplain die Tuffbank sofort um 200-300 m ansteigt. Eventuell sind tektonische Vorgänge an diesem Steilabfall zur Peneplain mitbeteiligt (s.Kap.2.1). Wir können uns aber durchaus auch vorstellen, dass es sich um einen erosiv und denudativ herauspräparierten Abhang randlich einer Einebnungsflạ̈che handelt(BUEDEL 1971, S.14). Dazu eignet sich arides oder semiarides Klima, wie es bereits im Pliozän hier vorgelegen hat. DOBROVOLNY (1962, S.30 f.) führt als weiteres Argument in die Diskussion, dass im Tal des Río Kaluyo, direkt unter dem Tuff, stark verkittete Ablagerungen vorkommen, die er als altpleistozäne Moränen bezeichnet. Diese "Drift Patapatani" weist aber weder gekritzte noch kantengerundete Steine auf und gibt somit keine schlüssigen Hinweise auf Moränenmaterial. Die starke Verkittung kann darin begründet liegen, dass dieser grobe Schotter in erhöhter Lage gerade ausserhalb des Wirkungsbereichs der Einebnungsvorgänge angehäuft worden ist. Er hat jedenfalls mehr oder weniger ungestört an der Oberfläche gelegen und ist dann vom Tuff überdeckt worden. Falls dieser Schotter altpleistozän sein sollte, so müsste der Steilabfall von $200-300 \mathrm{~m}$ rein tektonisch in einer darauffolgenden Zeit angelegt worden sein. Es ist aber recht unwahrscheinlich, dass eine solche tektonische Terrassenbildung nicht gemeinsam mit der mindestens $1000 \mathrm{~m}$ betragenden Hebung des Andenblocks (s.Kap.2.1.) erfolgt ist, oder dass all dies erst im Altpleistozän bzw. einem kurzen, verbleibenden Zeitraum vor sich gegangen ist.Es spricht also vieles dafür, dass die Peneplain mit ihrem randlichen Steilanstieg bereits am Ende des Oberpliozäns angelegt gewesen ist. Der Tuff markiert mit grosser Wahr scheinlichkeit die Pliozän/Pleistozängrenze.

\section{Genese}

Gestützt auf die erwähnten Datierungen versuchen wir nun, die Entstehung des Gebiets von La $\mathrm{Paz}$ zu umreissen. Im Laufe der andinen Orogenese, hauptsächlich zwischen Mittelmiozän und Oberpliozän (nach AHLFELD 1972, S.93), entstand das Altiplano als $200-300 \mathrm{~km}$ breite Depression zwischen der West- und Zentralkordillere. Mit der Hebung des Andenblocks ging eine intensive $\mathrm{Ge}-$ röllschüttung ins Altiplano einher, die aufgrund der beschriebenen Fossilien bis im Oberpliozän andauerte. Die Schotter der Formation La Paz erreichen 500-600 m Mächtigkeit (AHLFELD 1972, S.93). Gegen Ende des Oberpliozäns stellte sich ein gewisses Gleichgewicht zwischen Erosion, Akkumulation und Denudation ein, das zur Einebnung des Altiplano führte. Nach BUEDEL (1971, S.14/15) vollziehen sich solche Prozesse in der ariden Zone der Flächen-Erhaltung und -Ueberprägung sowie der Fussflächenbildung bzw. Pedimentation. Das primäre Altiplano stellte eine Peneplain dar, die im Gebiet der heutigen Stadt La Paz Höhen von 3850-3900 m erreichte. Als morphogenetische Grenze zwischen Altiplano und Zentralkordillere entstand hier, wahrscheinlich im ausgehenden Oberpliozän, eine $200-300 \mathrm{~m}$ hohe Steilstufe aus Schotter. Diese Nahtstelle war allenfalls tektonisch vorgeformt (AHLFELD 1972 , S.123/124). Heute verläuft sie, durch jüngeres Akkumulationsmaterial verdeckt, knapp unterhalb der Endmoränenwälle am Stadtrand von La Paz. Ueberraschenderweise bildete sich die Peneplain ziemlich ungestört, trotz der nachgewiesenen pliozänen tektonischen Bewegungen. Offenbar handelte es sich im Miozän und Pliozän vor allem um eine Hebung (LOHMANN 1970, S.754), die den Altiplanoblock ziemlich gleichmässig erfasste und höchstens den Andenblock etwas verstärkt hob. Daher verläuft die durch den Tuff konservierte Peneplain mehr oder weniger horizontal (TROLL \& FINSTERWALDER 1935, S.449). An ihrem Rand im heutigen Chuquiaguillotal wurde sie dann im Pleistozän lokal um ca. $100 \mathrm{~m}$ relativ abgesenkt (im Vergleich zum restlichen Altiplano, s. Fig.4). Ohne diese Dislokation müsste also der Tuffaufschluss unmittelbar westlich des Endmoränenwalls im Chuquiaguillotal ca. $100 \mathrm{~m}$ höher liegen, auf ca. 4150 m.ü.M. wie im benachbarten Tal des Río Kaluyo (s. TROLL \& FINSTERWALDER 1935, S.449).

Ebenfalls im Zusammenhang mit Dislokationen kann eine Reihe von fünf Seen nordwestlich der untersten Endmoränenwälle beim Río Kaluyo erklärt werden (16025'30'S/6809' W, $4270-4310 \mathrm{~m}$ ). Sie machen den Anschein von linear angeordneten Lösungshohlformen in Gips (mdl. Mitt. LOHMANN). Dieses Material befindet sich typischerweise an tektonischen Schwächezonen (AHLFELD 1946, S.243).

Während des Pleistozäns wurden Schotter und Moränen abgelagert 4 ). Ihre totale Mächtigkeit beträgt bei der morphogenetischen Altiplanogrenze $400-500 \mathrm{~m}$ und nimmt mit der Entfernung von der Zentralkordillere ab. Die untersten Glazialablagerungen sind auf dem Altiplano nordwestlich La Paz auf $3950 \mathrm{~m}$ feststellbar, reichen aber wohl gegen $100 \mathrm{~m}$ tiefer. Sie sind teilweise durch junge Schuttfächer überdeckt. Der optimale Schüttungswinkel für glaziale bzw. fluvioglaziale Ablagerungen ist in Fig. 3 mit der Altiplanokante verlässlich nachgezeichnet. Die pleistozänen Schüttungen stehen damit in deutlichem Kontrast (d.h. Diskordanz) zur Peneplain, welche durch den Tuff markiert ist. Die hauptsächliche Eintiefung der Schlucht vollzog sich nach diesen glazialen/fluvioglazialen Schüttungen, also frühestens seit dem letzten Interglazial. Die letzteiszeitlichen Gletscher bewegten sich indessen in bereits genau modellierten Tälern, und ihre Schotter wurden hauptsächlich unterhalb 4000 m.ü.M. geschüttet. Folglich geschah die Hauptausräumung des Talkessels'von La Paz vor der letzten Eiszeit, also im letzten Interglazial. Eine gewisse Ausräumung und Wiederauf-

\footnotetext{
4) Es sind mindestens zwei Eiszeiten unterscheidbar. Die Anzahl Eiszeiten ist aber kaum genau festlegbar, da keine ausreichenden interglazialen Bodenbildungen anzutreffen sind. SERVANT (1977, S.324) erwähnt zwar solche im Raume La Paz und hat parallel dazu mehrere interglaziale Einebnungsflächen studiert.
} 
Foto 7 (Farbfoto Albert Leemann, April 1967) Blick vom Altiplanorand auf die Stadt La $\mathrm{Paz}$ und die vergletscherten Gipfel des Huayna Potosi (6088 m, links) und Chacaltaya (5395 $\mathrm{m}$, rechts). Im Vordergrund befinden sich fluvioglaziale Schotter der vorletzten Eiszeit. Der kleine Weg führt zum Hauptfriedhof von La Paz hinunter (rechts, mit Eukalyptusbäumen). In der Bildmitte erkennt man die Hauptstrasse in der Talachse. Dahinter erstreckt sich der Stadtteil Achachicala (s.Fig.3), dessen oberste Häuser auf $3850 \mathrm{~m}$.ü.M. ungefähr die Höhenlage der Tuffschicht abstecken. Hinter Achachicala sieht man den Rio Kaluyo und einen gewellten Talabschnitt in der Sonne, die vordersten Endmoränen aus der letzten Eiszeit.

schotterung erfolgte sicher schon früher, erlangte aber am heutigen Relief keinen entscheidenden Einfluss, weil die Prozesse im letzten Interglazial sie weit übertrafen.

Es ist kaum nachweisbar, warum sich der Talkessel genau an seinem jetzigen Ort eingetieft hat. Zufällig fand das Wasser aus der relativ feuchten Zentralkordillere hier am leichtesten einen Abfluss und brachte intensive Erosions- und Denudationsprozesse in Gang. Die Flüsse fanden hier ihren nächsten und direkten Weg quer durch die Zentralkordillere. Vielleicht wurde der Abtrag durch das Wasser im letzten Interglazial durch die letzten Hebungsphasen des Andenblocks (AHLFELD 1972, S.95/96) verstärkt. AHLFELD korrigiert in dieser Arbeit seine frühere Aeusserung (AHLFELD 1946, S.95), wonach das Alter der Schlucht von La Paz ausschliesslich postglazial sei. Natürlich erfolgte im heutigen Stadtgebiet im Postglazial eine weitere Eintiefung. Sie wird bis jetzt fortgesetzt, abwechselnd mit Hangrutschungen und Murgängen. Am geringsten ist diese Ueberprägung in den Talabschnitten oberhalb 4000 $\mathrm{m}$, da dort die erosionsanfälligen letzteiszeitlichen Schotter praktisch fehlen und das Klima noch nicht arid ist wie weiter talabwärts. Demgegenüber werden die letzteiszeitlichen Schotterterrassen im Talboden von La Paz badlandsartig zerschnitten und oft erneut von Hangrutschmassen überdeckt. Die letzten grossen Rutschungen im Februar 1974 erfassten zahlreiche Häuser und Hausgruppen. Sie haben in katastrophaler Art gezeigt, wie aktiv auch heute noch die geomorphologischen Prozesse im Talkessel von La Paz sind.

\section{Summary}

The town of La Paz is situated between the Bolivian Highland (Altiplano) and the Central Mountain Range. These two landscape units were mostly formed during the Tertiary. But at their intermediate bordering zone, geomorphological forms have later been reshaped. At the end of the Pliocene, a peneplain was formed (the Altiplano of today) and got covered by volcanic ashes. Since the fossilization of mammals (Pos- nanskytherium) in the late Pliocene, a tektonical uplift of about $3300 \mathrm{ft}$. has taken place, and huge moraines built up covering the whole city area of today. The enormous La Paz Valley was eroded above all during the last interglacial period. During the last ice age, the glaciers reached the present upper city border only and melted away very rapidly 9800 years ago at the latest.

\section{Literatur}

AHLFELD, F.:Geología de Bolivia. Revista del Museo de La Plata, Rep. Argentina (Nueva Serie), Sección Geología III, 1946, S.5-370.

" Geología de Bolivia. Ed. "Los Amigos del Libro", La Paz, 1972, S.7-190.

BUEDEL, J.: Das natürliche System der Geomorphologie. Würzburger Geogr. Arb., H.34, 1971, S.1-152.

CLOCCHIATTI, R., LERIBAULT, L. \& RODRIGO, L.A. : Endoscopie et exoscopie de grains de quartz des formations du Pliocène et du Quaternaire de La Paz (Bolivie). Cah. O.R.S.T.O.M., Sér. Géol., Vol.X, no.1, 1978, S.127-143.

DOBROVOLNY, E.: Geología del Valle de La Paz. Min. de Minas y Petróleo, Dep. Nac. de Geología, Boletín No.3 (Especial), La Paz,1962, S. 4-153.

EVERDEN, J.F., KRIZ, S.J. \& CHERRONI, C. : Potassium-Argon ages of some Bolivian rocks. Economic Geology, Vol.72, No.6, 1977, S.10421061 .

GRAF, K.: Estudios Geomórficos en los Andes y el Altiplano Bolivianos. Soc. Geol. Boliv., Boletín No.21, 1975, S.3-23.

LOHMANN, H.H.: Outline of Tectonic History of Bolivian Andes. The American Ass. of Petroleum Geol. Bull., Vol.54, No.5, 1970, S.735757.

NEVELL, N.D.: Geology of the Lake Titicaca Region, Peru and Bolivia. Geol. Soc. of America, New York, 1949, Mem. 36.

POSNANSKY, A.: Apuntes antropogeológicos andinos. Bol. Soc. Geogr. de La Paz, Año 28, Nos. $51 \& 52$, 1920, S.93-106.

RADELLI, L.: Ensayo de reconstrucción de la cronoestratigrafía y de la paleogeografía del Altiplano y de las cordilleras orientales de Bolivia después del Paleozoico superior. Riv. Italiana Paleont., Vol.70, No. 4, 1964, S.833-868.

SERVANT, M.: Le cadre stratigraphique du PlioQuaternaire de 1'Altiplano des Andes tropicales en Bolivie. INQUA, Suppl.au Bulletin AFEQ, Bd.1., No.50, 1977, S.323-327.

TROLL, C. \& FINSTERWALDER, R.: Die Karten der Cordillera Real und des Talkessels von La Paz (Bolivien) und die Diluvialgeschichte der zentralen Anden. Petermanns Geogr. Mitt., H. $11 \& 12,1935$, S.393-455.

VILLARROEL, C.: Revalidación y redescripción de Posnanskytherium desaguaderoi Liendo, 1943; Toxodontidae (Notoungulata) del Plioceno superior boliviano. Boletín del Servicio Geológico de Bolivia, Serie A, Vol.I, No.1, 1977, S. 21-32. 